\title{
Roughening and super-roughening in the ordered and random two-dimensional sine-Gordon models
}

\author{
Angel Sánchez* \\ Grupo Interdisciplinar de Sistemas Complicados (GISC), Departamento de Matemáticas, Universidad Carlos III de Madrid, \\ Avenida de la Universidad 30, 28911 Leganés, Madrid, Spain
}

A. R. Bishop

Theoretical Division and Center for Nonlinear Studies, MS B258, Los Alamos National Laboratory, Los Alamos, New Mexico 87545

Esteban Moro

Theoretical Physics Department, University of Oxford, 1 Keble Road, Oxford OX1 3NP, United Kingdom

(Received 10 March 2000)

\begin{abstract}
We present a comparative numerical study of the ordered and the random two-dimensional sine-Gordon models on a lattice. We analytically compute the main features of the expected high-temperature phase of both models, described by the Edwards-Wilkinson equation. We then use those results to locate the transition temperatures of both models in our Langevin dynamics simulations. We show that our results reconcile previous contradictory numerical works concerning the super-roughening transition in the random sine-Gordon model. We also find evidence supporting the existence of two different low-temperature phases for the disordered model. We discuss our results in view of the different analytical predictions available and comment on the nature of these two putative phases.
\end{abstract}

PACS number(s): 05.10.Gg, 68.35.Ct, 74.60.Ge, 64.70.Pf

\section{INTRODUCTION}

The location and characteristics of phase transitions in disordered media constitute a long-standing and controversial question, particularly in more than one spatial dimension [1]. The question becomes even more difficult if the disorder is not very weak; then, new, nontrivial behavior is commonly found, involving features such as aging, ergodicity breaking, extremely slow dynamics, complicated energy landscapes, etc.; major examples of this are spin glasses and structural glasses $[1,2]$. In this context, the properties of crystal surfaces growing on disordered substrates, frequently described by a two-dimensional random-phase sine-Gordon model (RSGM), have attracted a lot of attention in the past decade [3-22]. Interestingly, the same model describes many other relevant physical problems, such as randomly pinned flux lines confined in a plane [5,21,23-25], vortex lines in planar Josephson junctions [26], charge-density waves [27], and commensurate-incommensurate transitions [28].

In spite of those efforts, the phase diagram and main features of the RSGM are not clear yet. To summarize what is known, we refer for comparison to the ordered sine-Gordon model (OSGM), which is rather well understood (see, e.g., $[1,29-33]$ and references therein). The Hamiltonian for the OSGM and the RSGM is

$$
\begin{aligned}
H= & \sum_{r}\left(\frac{1}{2} \sum_{n n}\left[h(\mathbf{r})-h\left(\mathbf{r}^{\prime}\right)\right]^{2}\right. \\
& \left.+V_{0}\left\{1-\cos \left[h(\mathbf{r})-h^{(0)}(\mathbf{r})\right]\right\}\right),
\end{aligned}
$$

\footnotetext{
*Electronic address: anxo@math.uc3m.es; URL: http://valbuena.fis.ucm.es/ anxo
}

where nn stands for the nearest neighbors of site $\mathbf{r}$. The OSGM corresponds to $h^{(0)}(\mathbf{r}) \equiv 0$, whereas the RSGM is defined by choosing the quenched disorder variables $h^{(0)}(\mathbf{r})$ randomly from a uniform distribution in $[0,2 \pi]$. We will discuss our work in terms of growth on flat (OSGM) or disordered (RSGM) substrates (see [24-28] for other physical interpretations): Accordingly, $h(\mathbf{r})$ is a continuous variable representing the height of the growing surface at site $\mathbf{r}$ of the lattice, and the cosine term is a potential energy making integer (i.e., multiples of the crystalline lattice constant) values of the height energetically favorable. We will consider twodimensional (2D) square lattices, i.e., $\mathbf{r}=\left(r_{1}, r_{2}\right)$, with $N$ $=L \times L$ sites. As first shown by Chui and Weeks [34] (see also [35]) by means of a renormalization-group (RG) approach [36], the OSGM possesses a Kosterlitz-Thouless [37] type topological transition between a low-temperature, flat phase and a high-temperature, rough phase, the latter being described by the Edwards-Wilkinson (EW) equation [38], i.e., the diffusion equation with additive white noise (see below). Above the so-called roughening temperature $\left(T_{R}\right)$, thermal fluctuations effectively suppress the effect of the cosine potential, and the surface becomes free, described only by the kinetic part of the Hamiltonian (1). As will be shown below, the most important measurable consequence of this is that the width of the interface,

$$
w^{2}=\frac{1}{N} \sum_{r}[h(\mathbf{r})-\bar{h}]^{2}, \quad \bar{h}=\frac{1}{N} \sum_{r} h(\mathbf{r}),
$$

scales (in $2 \mathrm{D}$ ) as $w^{2} \sim \ln L$ in the asymptotic regime.

In contrast with the clear picture for the OSGM, there are very few generally accepted results for the RSGM. One of them is that there must be a roughening temperature above which the potential effectively vanishes (much as in the case 
of the OSGM) leading to EW behavior. Apart from this, theoretical predictions about the low-temperature phase largely disagree (a good summary is given in the third paper in Ref. [7]): While RG calculations predict a super-rough low-temperature phase, with $w^{2} \sim \ln ^{2} L$, replica-symmetry breaking variational approaches lead to $w^{2} \sim \ln L$ independently of temperature. Numerical simulations were not very conclusive either: Batrouni and Hwa [5] did not find evidence for an equilibrium phase transition in Langevin dynamics, although Monte Carlo simulations by Rieger [6] showed a transition from a super-rough phase to an EW phase for stronger potentials [larger $V_{0}$ in Eq. (1)] than those used by Batrouni and Hwa. Subsequent numerical work $[11,12,16,18]$ presented more evidence of super-rough $\left(\ln ^{2} L\right)$ behavior, albeit with large quantitative discrepancies with the predictions of RG theories. Finally, a number of works using special optimization algorithms $[13,15,19]$ or direct numerical simulations [17] strongly supported superrough behavior at zero temperature. Very recently [21], simulations of a related model provided more evidence of $\ln ^{2}$ behavior at finite temperatures, although this model did not allow study of the transition. In summary, most researchers believe that there is indeed a super-rough low-temperature phase in the RSGM, but its nature (glassy or not), the transition temperature, and its dependence on the model parameters remain unclear.

In this paper, we attempt to shed light on these issues by simultaneously studying the OSGM and the RSGM in different regions of their phase diagram. As we will show below, it turns out that the potential strength, $V_{0}$, crucially determines the model features. In addition, it is also natural to ask about the intensity of the disorder. How does the model phenomenology change if the disorder takes values in $[0, \epsilon]$ with $\epsilon<2 \pi$ ? The importance of these points can be clearly seen in the Langevin equation,

$$
\frac{d h(\mathbf{r}, t)}{d t}=-\frac{\delta H}{\delta h(\mathbf{r}, t)}+\eta(\mathbf{r}, t),
$$

where $\eta(\mathbf{r}, t)$ is a Gaussian white noise of zero mean and correlations obeying the fluctuation-dissipation theorem,

$$
\left\langle\eta\left(\mathbf{r}^{\prime}, t^{\prime}\right) \eta(\mathbf{r}, t)\right\rangle=2 T \delta^{(2)}\left(\mathbf{r}-\mathbf{r}^{\prime}\right) \delta\left(t-t^{\prime}\right),
$$

where $T$ stands for the temperature of the system, $\langle\cdots\rangle$ indicates thermal averages (over $\eta$ ), and Boltzmann's constant is set to $k_{B}=1$. For our Hamiltonian, Eq. (1), the Langevin equation is

$$
\frac{\partial h(\mathbf{r}, t)}{\partial t}=\nabla^{2} h(\mathbf{r}, t)-V_{0} \sin \left[h(\mathbf{r}, t)-h^{(0)}(\mathbf{r})\right]+\eta(\mathbf{r}, t),
$$

and changing variables according to $\widetilde{h}(\mathbf{r}, t)=h(\mathbf{r}, t)$ $-h^{(0)}(\mathbf{r})$ (i.e., the height referred to the substrate), we find

$$
\frac{\partial \widetilde{h}(\mathbf{r}, t)}{\partial t}=\epsilon F(\mathbf{r})+\nabla^{2} \widetilde{h}(\mathbf{r}, t)-V_{0} \sin (\widetilde{h}(\mathbf{r}, t))+\eta(\mathbf{r}, t),
$$

where $F(r)=\nabla^{2} h^{(0)}(\mathbf{r}) / \epsilon$. In this form, the disorder is a random (correlated in space) chemical potential acting on a surface growing on a flat substrate. If we think of the roughening transition for the OSGM in terms of the interplay between the temperature $T$ and the energy scale introduced by the potential, $V_{0}$, when $\epsilon \neq 0$, we have another energy scale, $\epsilon$, which can modify the universal features of the roughening transition or even give rise to novel thermodynamical transitions.

Having the above issues in mind, we discuss our work according to the following scheme. Section II presents an analytical study of the EW equation and other statistical mechanics results about the energy and roughness. Section III deals with the main part of our work, namely Langevin dynamics simulations of the OSGM and the RSGM, beginning with $V_{0}=1$ and $\epsilon=2 \pi$ and subsequently analyzing the model behavior for different $V_{0}$ and $\epsilon$. Finally, in Sec. IV we present and discuss our conclusions and indicate future directions.

\section{ANALYTICAL RESULTS}

\section{A. Linear theory: The Edwards-Wilkinson equation}

According to the RG approach [29], the high-temperature phase of the OSGM obeys the EW equation [38],

$$
\frac{\partial h(\mathbf{r}, t)}{\partial t}=\nabla^{2} h(\mathbf{r}, t)+\eta(\mathbf{r}, t) .
$$

Equation (7) can be solved by means of the Fourier decomposition (see [32] for details),

$$
\hat{h}_{\mathbf{q}}=\frac{1}{L} \sum_{r} e^{i \mathbf{q} \cdot \mathbf{r}} h(\mathbf{r}, t),
$$

where $\mathbf{q}=(2 \pi / L) \mathbf{k}, k_{i}=0, \ldots, L-1$ is the reciprocal vector. The structure factor can then be shown to be

$$
S(\mathbf{q})=\left\langle\hat{h}_{\mathbf{q}} \hat{h}_{-\mathbf{q}}\right\rangle=T \frac{1-e^{-2 \omega_{\mathbf{q}} t}}{\omega_{\mathbf{q}}},
$$

$\omega_{\mathbf{q}}$ being the $2 \mathrm{D} \mathrm{EW}$ discrete dispersion relation

$$
\omega_{\mathbf{q}}=4 \sin ^{2}\left(\frac{q_{1}}{2}\right)+4 \sin ^{2}\left(\frac{q_{2}}{2}\right) .
$$

From $S(\mathbf{q})$ we can obtain the relevant magnitudes, such as the total roughness,

$$
w^{2}(t)=\left\langle\frac{1}{L^{d}} \sum_{\mathbf{r}}[h(\mathbf{r}, t)-\bar{h}]^{2}\right\rangle=\frac{1}{L^{d}} \sum_{\mathbf{q} \neq \mathbf{0}} S(\mathbf{q}),
$$

the correlation function,

$$
\begin{aligned}
C(\mathbf{r}) & =\left\langle\frac{1}{L^{2}} \sum_{\mathbf{s}}[h(\mathbf{s}+\mathbf{r})-h(\mathbf{s})]^{2}\right\rangle \\
& =\frac{2}{L^{2}} \sum_{\mathbf{q}} S(\mathbf{q})[1-\cos (\mathbf{q} \cdot \mathbf{r})],
\end{aligned}
$$

the total slope,

$$
s^{2}(t)=\frac{1}{L^{2}} \sum_{\mathbf{q} \neq \mathbf{0}} S(\mathbf{q}) \omega_{\mathbf{q}}
$$


and the slope difference correlation function,

$$
\begin{aligned}
G(\mathbf{r}) & =\left\langle\frac{1}{L^{2}} \sum_{\mathbf{s}}[\boldsymbol{\nabla} h(\mathbf{s}+\mathbf{r})-\boldsymbol{\nabla} h(\mathbf{s})]^{2}\right\rangle \\
& =\frac{4}{L^{2}} \sum_{\mathbf{q}} S(\mathbf{q}) \sum_{i=1}^{2}\left[1-\cos \left(q_{i} r_{i}\right)\right]\left[1-\cos \left(q_{i}\right)\right] .
\end{aligned}
$$

From Eq. (9) we can find an estimate for the time needed to reach saturation, $t_{x}$, and the dynamic exponent, $z$ : We compute the time that the structure factor needs to be within $1 \%$ of its saturated form for the slowest Fourier mode, that with the lowest $|\mathbf{q}|,|\mathbf{q}|=2 \pi / L$ obtaining

$$
t_{\times} \simeq 3 \times 10^{-2} L^{2}
$$

implying $z=2$. For the saturated roughness, we obtain

$$
w^{2}(t \rightarrow \infty, L)=\frac{T}{4 L^{2}} \sum_{k_{1}, k_{2}=1}^{L-1}\left[\sin ^{2}\left(\frac{q_{1}}{2}\right)+\sin ^{2}\left(\frac{q_{2}}{2}\right)\right]^{-1},
$$

which cannot be computed exactly but, for large $L$, can be approximated changing the sum by an integral and the sine functions by their arguments, arriving at

$$
\begin{aligned}
w^{2}(t \rightarrow \infty, L) & \simeq \frac{1}{(2 \pi)^{2}} \int_{2 \pi / L}^{\pi} \int_{2 \pi / L}^{\pi} d q_{x} d q_{y} \frac{T}{\left(q_{x}^{2}+q_{y}^{2}\right)} \\
& \simeq \frac{T}{2 \pi} \int_{2 \pi / L}^{\pi} \frac{d q}{q}=\frac{T}{2 \pi} \ln L
\end{aligned}
$$

yielding a roughness exponent $\alpha=0$. As for the total slope, it tends to a value independent of $L$,

$$
s^{2} \simeq T
$$

whereas for the correlation functions, we find for large $r$

$$
C(\mathbf{r}) \simeq \frac{T}{\pi} \ln r
$$

$G(\mathbf{r}) \simeq 2 s^{2}+\frac{T}{4 \pi} \ln \frac{r^{8}}{\left[\left(r^{2}+1\right)^{2}-4 r_{1}^{2}\right]\left[\left(r^{2}+1\right)^{2}-4 r_{2}^{2}\right]} \simeq 2 T$

\section{B. Other results for the energy and the roughness}

At equilibrium, the partition function for the OSGM is

$$
Z=\int\left[\prod_{\mathbf{r}} d h(\mathbf{r})\right] e^{-\beta\left\{(1 / 2) \Sigma_{\mathbf{r}}(\boldsymbol{\nabla} h(\mathbf{r}))^{2}+V_{0}[1-\cos h(\mathbf{r})]\right\}},
$$

where $\beta=T^{-1}$. Expanding for high temperatures [41] means rewriting Eq. (21) as a series in powers of $\beta V_{0}$ :

$$
\begin{aligned}
Z= & \sum_{n=0}^{\infty} \frac{\left(\beta V_{0}\right)^{n}}{n !} \int\left[\prod_{\mathbf{r}} d h(\mathbf{r})\right] e^{-\beta \Sigma_{\mathbf{r}}(1 / 2)(\nabla h(\mathbf{r}))^{2}} \\
& \times\left(\sum_{\mathbf{r}} \cos h(\mathbf{r})\right)^{n} \\
= & Z_{0} \sum_{n=0}^{\infty} \frac{\left(\beta V_{0}\right)^{n}}{n !}\left\langle\left(\sum_{\mathbf{r}} \cos h(\mathbf{r})\right)^{n}\right\rangle_{H_{0}},
\end{aligned}
$$

where $Z_{0}$ is the partition function of $H_{0}$, the free Hamiltonian [i.e., Eq. (1) without the potential term]. By means of this expansion we obtain

$$
\begin{aligned}
\left\langle\cos h\left(\mathbf{r}^{\prime}\right)\right\rangle_{H}= & \frac{Z_{0}}{Z} \sum_{n=0}^{\infty} \frac{\left(\beta V_{0}\right)^{n}}{n !} \\
& \times\left\langle\left(\sum_{\mathbf{r}} \cos h(\mathbf{r})\right)^{n} \cos h\left(\mathbf{r}^{\prime}\right)\right\rangle_{H_{0}},
\end{aligned}
$$

where subdominant terms such as $\beta^{n} \exp (-A / \beta)$ have been neglected, and only terms of the order $\beta^{n}$ have been kept. The expression above can be put in the form

$$
\begin{aligned}
\left\langle\cos h\left(\mathbf{r}^{\prime}\right)\right\rangle_{H} & =\left(\sum_{n=1}^{\infty} \frac{2^{2 n-1}(n !)^{2}}{n\left(\beta V_{0}\right)^{2 n-1}}\right) \times\left(\sum_{n=0}^{\infty} \frac{\left(\beta V_{0}\right)^{2 n}}{2^{2 n}(n !)^{2}}\right)^{-1} \\
& =\frac{\beta V_{0}}{2}-\frac{\left(\beta V_{0}\right)^{3}}{16}+\frac{\left(\beta V_{0}\right)^{5}}{96}+O\left(\left(\beta V_{0}\right)^{7}\right),
\end{aligned}
$$

yielding for the approximate energy per site

$$
E=\frac{1}{L^{2}}\langle H\rangle=\frac{1}{2 \beta}+V_{0}\left(1-\frac{\beta V_{0}}{2}+\frac{\left(\beta V_{0}\right)^{3}}{16}+O\left(\left(\beta V_{0}\right)^{5}\right)\right) \text {. }
$$

For the roughness, we have to compute

$\left\langle[h(\mathbf{r})-\bar{h}]^{2}\right\rangle_{H}=\frac{Z_{0}}{Z}$

$$
\times \sum_{n=0}^{\infty} \frac{\left(\beta V_{0}\right)^{n}}{n !}\left\langle h^{2}(\mathbf{r}) \sum_{\mathbf{r}^{\prime}}\left[\cos h\left(\mathbf{r}^{\prime}\right)\right]^{n}\right\rangle_{H_{0}}
$$

assuming that at equilibrium $\bar{h}=0$. Neglecting again subdominant terms, we find

$$
\left\langle h^{2}(\mathbf{r})\right\rangle_{H}=\frac{Z_{0}}{Z}\left\langle h^{2}(\mathbf{r})\right\rangle_{H_{0}}
$$

and hence

$$
w_{H}^{2}=w_{H_{0}}^{2}\left(1-\frac{\beta V_{0}}{4}+\frac{3\left(\beta V_{0}\right)^{2}}{64}-\frac{19\left(\beta V_{0}\right)^{3}}{2304}+O\left(\left(\beta V_{0}\right)^{4}\right)\right)
$$

Finally, at low temperatures the height exhibits only small deviations from $h=0$, and therefore we can approximate the Hamiltonian as 


$$
\begin{aligned}
H & =\sum_{i} \frac{1}{2}(\boldsymbol{\nabla} h(\mathbf{r}))^{2}+V_{0}[1-\cos (h(\mathbf{r}))] \\
& \simeq \sum_{i} \frac{1}{2}(\boldsymbol{\nabla} h(\mathbf{r}))^{2}+V_{0} \frac{h(\mathbf{r})^{2}}{2}
\end{aligned}
$$

i.e., $2 N$ quadratic terms, each one of which, according to the equipartition theorem [42], contributes with $T / 2$ to the energy value. Taking into account the global factor $\frac{1}{2}$, we conclude that at low temperatures the energy of the OSGM is approximately $E=T / 2$.

\section{LANGEVIN DYNAMICS RESULTS}

\section{A. Numerical simulation details}

We have integrated the Langevin equation (5) corresponding to the Hamiltonian (1), on $L \times L$ square lattices with periodic boundary conditions, using a stochastic second-order Runge-Kutta method [43]; in some cases, we have repeated the simulations with a Heun method [44], with excellent agreement between both procedures. We are therefore sure that our results are not an artifact of our numerical method, a conclusion further reinforced by the agreement with the theoretical expectations of the preceding section as we discuss below. The simulations reported in this paper were carried out with a time step $\Delta t=0.01$ on lattices of sizes $L=64,128$, and 256. It is important to stress that we did not perform averages over the quenched noise in the RSGM; however, we checked that the outcome of our simulations did not depend strongly on the realization of the quenched noise or the initial conditions [flat, $h(\mathbf{r})=0$; as the substrate, $h(\mathbf{r})=h^{(0)}(\mathbf{r})$, or random] by repeating several times a number of our simulations. In all cases, simulations consisted of an equilibration time and a measuring period. Equation (15) predicts that the time needed for equilibration is $t_{\times} \simeq 500$ for $L=128$ and $t_{\times} \simeq 2000$ for $L=256$, and hence we used equilibration times of 5000 and 10000 units, respectively; afterwards, we let the system evolve for an equal period, over which we performed thermal averages. Equilibration was ensured in all cases by verifying that the fluctuations of the energy were Gaussian and by checking the equality of the specific heat computed from those fluctuations and from derivatives of the mean energy [1], as well as by monitoring the evolution in time of the quantities of interest toward a stationary state. As an additional test, we compared the imposed simulation temperature, arising from the noise term, to that measured during the evolution according to the equipartition theorem [42]; both quantities were always found to agree within $0.1 \%$. Finally, we did a few much longer runs, whose outcome agreed with that of the shorter runs.

\section{B. Standard RSGM: $\quad V_{0}=1$ and $\epsilon=2 \pi$}

We begin by discussing our results for the "canonical", version of both the OSGM and the RSGM, i.e., Hamiltonian (1) with $V_{0}=1$, as studied (for the ordered case) in $[39,40]$. In those works, the roughening temperature was determined by a direct comparison to RG predictions, looking for the temperature at which the height difference correlation function reached a universal (in the RG framework) value, with

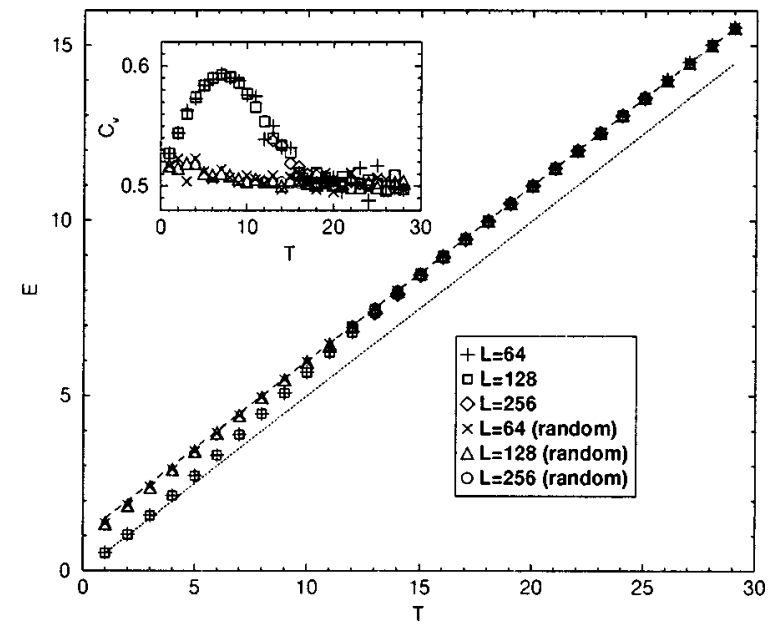

FIG. 1. Mean energy for the OSGM and the RSGM vs temperature. Symbols as indicated in the plot. The straight lines correspond to the low-temperature prediction (lower line) and the hightemperature prediction (upper line). Inset: Specific heat vs temperature; symbols as in the main plot. Error bars are smaller than the symbol size.

the result that for the OSGM $T_{R} \approx 25$ in our dimensionless units. Remarkably, this is the RG value for $T_{R}$, which makes it very tempting to claim that this method indeed yields $T_{R}$ correctly. Monte Carlo simulations of the discrete Gaussian model [Hamiltonian (1) with $V_{0}=0$ and $h(\mathbf{r})$ restricted to integer values] by Shugard et al. [45] with the same criterion for locating the transition yielded similar results. However, as RG calculations are perturbative in $V_{0}$ and carried out on the continuum Langevin equation [29-31,33-35], it is not obvious that they apply to a discrete model with $V_{0}=1$, i.e., of the same order as the kinetic term. In view of this, we decided to include the OSGM in this study, both to analyze in detail whether the comparison to the universal RG prediction for the factor is a good tool to find $T_{R}$ and to compare its high-temperature phase with that of the RSGM, which should also be of EW type.

The first quantity we discuss, shown in Fig. 1, is the mean energy of both models. As we see, the results are largely independent of the system size, and hence it is unlikely that they are affected by finite-size effects. The plot shows that the mean energy of the OSGM reaches the high-temperature approximation at $T_{0}=16 \pm 1$; on the other hand, the mean energy for the RSGM is never too far from it, although for temperatures lower than $T_{1}=4 \pm 1$ the numerical values lie slightly below the high-temperature result. At temperatures higher than $T_{0}$ the energies of both models coincide within the accuracy of our simulations. These results suggest that $T_{0}$ could be the roughening temperature, $T_{R}$, for the OSGM and $T_{1}$ the super-roughening temperature, $T_{\mathrm{SR}}$, for the RSGM, because the EW behavior of the mean energy of both models indicates the effective suppression of the sine term by temperature. The inset in Fig. 1 presents the specific heat, $C_{v}$, of both models, exhibiting a well defined peak in $C_{v}$ for the OSGM with its maximum at temperature $T=9$, much lower than $T_{0}$. In contrast, we do not observe any peak for the RSGM; there might be a peak at $T=3$, but the evidence is not conclusive. Concerning the peak for the OSGM, we stress again the absence of any finite-size effect, consistent 


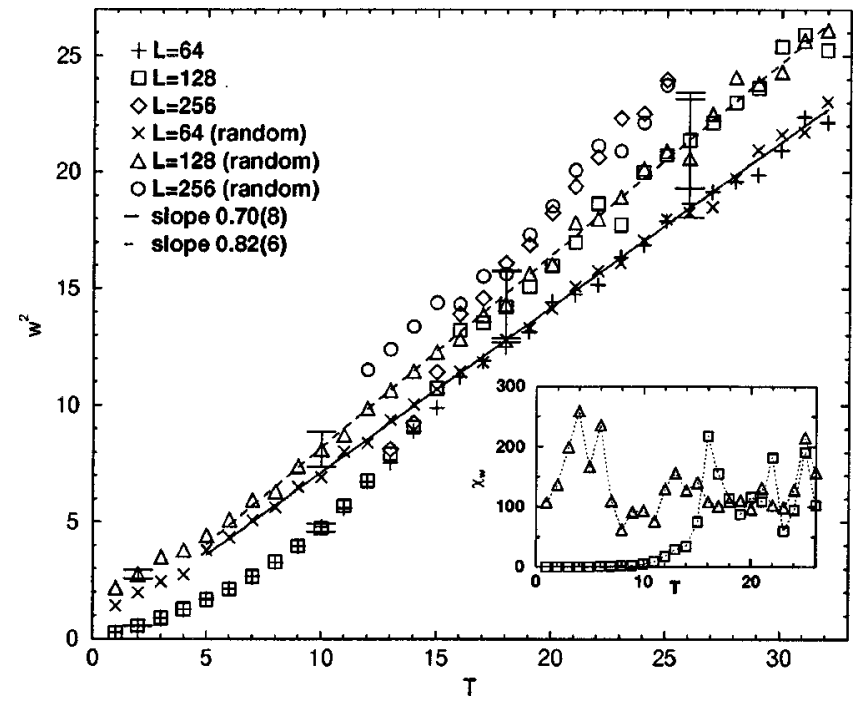

FIG. 2. Total roughness for the OSGM and the RSGM vs temperature. Symbols as indicated in the plot. The straight lines correspond fits (resulting slopes are also given in the plot) to the hightemperature prediction for the two sizes considered. Inset: Roughness susceptibility vs temperature; symbols as in the main plot. Examples for error bars in different regions are shown.

with a KT-type transition. Furthermore, we believe that this peak is a (Schottky) anomaly [36] similar to that observed in 2D $X Y$ and easy-plane Heisenberg spin models [46] above the KT transition. Recall that when mapping the OSGM to the $X Y$ model, the temperature of the former maps to the inverse temperature of the latter [34], hence the observation of the anomaly below the possible transition temperature $T_{0}$. This reinforces our interpretation of $T_{0}$ as the roughening temperature of the OSGM.

The total roughness of both models, shown in Fig. 2, behaves similarly for temperatures higher than $T_{0}$, depending linearly on temperature. In both cases, we see that the slope of the roughness depends on the system size, as predicted by Eq. (16). Whereas the approximation in Eq. (17) yields slopes 0.66 and 0.77 for $L=64$ and 128 , respectively, if we numerically compute the exact result, Eq. (11), the slopes turn out to be 0.71 and 0.82 , in excellent agreement with the results of our simulations, 0.71 and 0.83 . Below $T_{0}$, the roughness for the OSGM is independent of the size and depends nonlinearly on temperature, whereas above $T_{0}$ we find a linear dependence on temperature and clear finite-size effects. For the RSGM, the linear behavior extends all the way down to $T_{1}$, and below $T_{1}$ the behavior becomes nonlinear. The slope of the linear region is approximately the same in the first part, from $T_{1}$ to $T_{0}$, and in the second, above $T_{0}$, i.e., the whole linear region is well described by the EW model. This means that above $T_{0}$ the linear model describes accurately the behavior of the OSGM, and hence from now on we identify $T_{0}$ with the roughening temperature $T_{R}$, whereas for the RSGM, the same is true of $T_{1}$ and $T_{\mathrm{SR}}$. Figure 2 presents also results for the roughness susceptibility, $\chi_{w}$, defined as $\chi_{w}^{2}=\left[\left\langle\left(w^{2}\right)^{2}\right\rangle-\left\langle w^{2}\right\rangle^{2}\right] / T^{2}$. For the OSGM, $\chi_{w}$ exhibits a very clear peak at $T_{R}$, and above $T_{R}$ it is the same as for the RSGM; however, this magnitude is very noisy and these results must be taken with caution. In fact, one could identify a peak for the RSGM at $T_{\mathrm{SR}}$, but
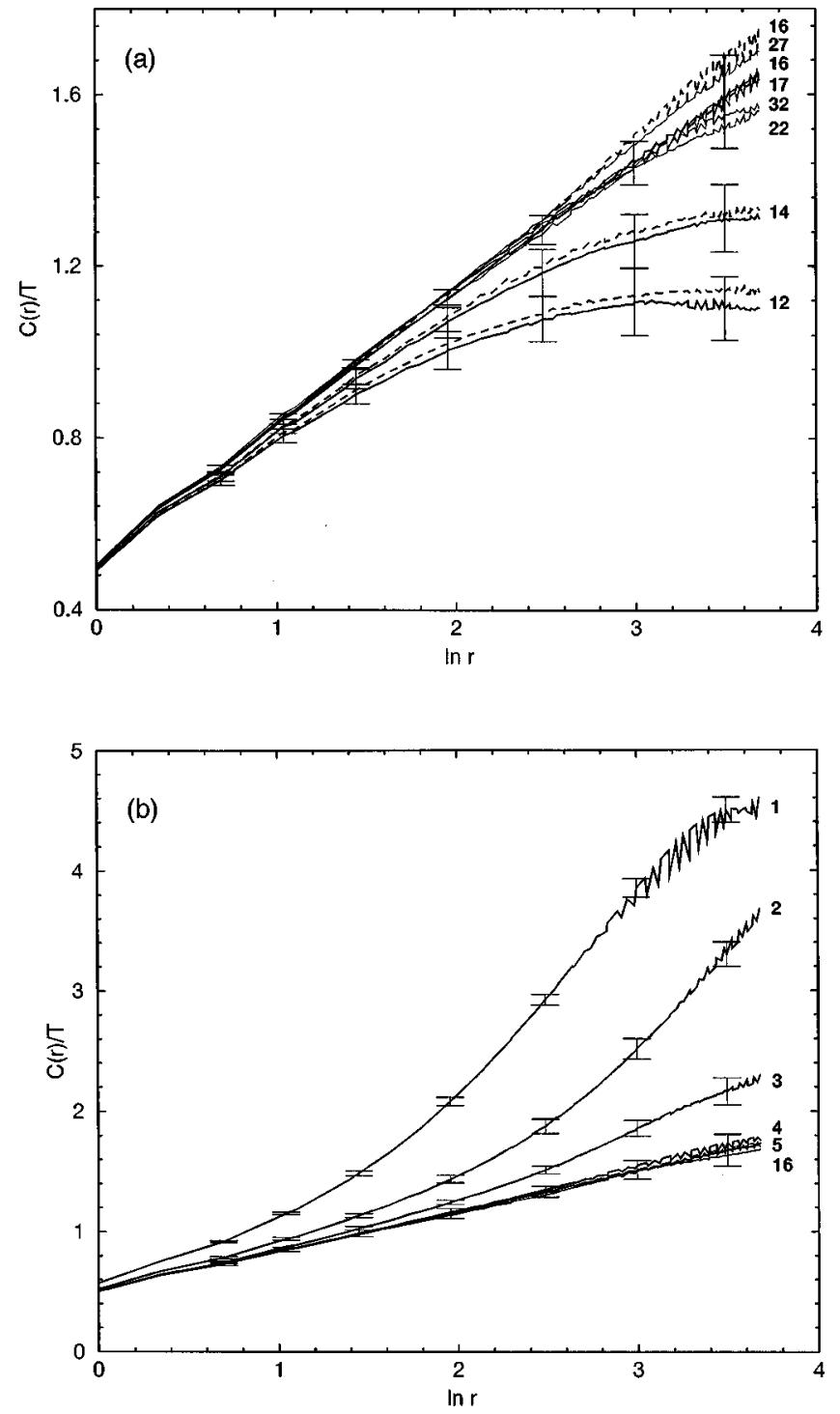

FIG. 3. Height difference correlation functions (scaled by $T$ ) vs $\ln r, r$ being distance, for the OSGM (upper panel) and disordered (lower panel) sine-Gordon model. Temperatures are indicated at the right side of the plots. The curve marked with temperature 16 in the plot for the RSGM is the correlation function for the OSGM at that temperature, showing clearly that both overlap.

different realizations lead to different results, in contrast with the peak for the OSGM, which is the same for all realizations. Below $T_{R}$, the values of $\chi_{w}$ for the OSGM are independent of the system size, whereas above $T_{R}$ they increase with size without any definite scaling.

Figure 3 depicts the height-difference correlation function for the two studied models, and shows that above $T_{R}=T_{0}$ and $T_{\mathrm{SR}}=T_{1}$ they behave as predicted by the linear theory: The slope of the numerical height-difference correlation function is 0.32 , indistinguishable from the predicted 0.318 by Eq. (19). In addition, the correlation functions for the OSGM and the RSGM coincide, as shown in the plot for the RSGM. We see that the behavior of the correlation functions is in full agreement with our claims regarding $T_{R}$ and $T_{\mathrm{SR}}$, and this is further confirmed by the plot of the slope correlation function in Fig. 4. It is important to note that, below $T_{\mathrm{SR}}$, the behavior of the height-difference correlation func- 

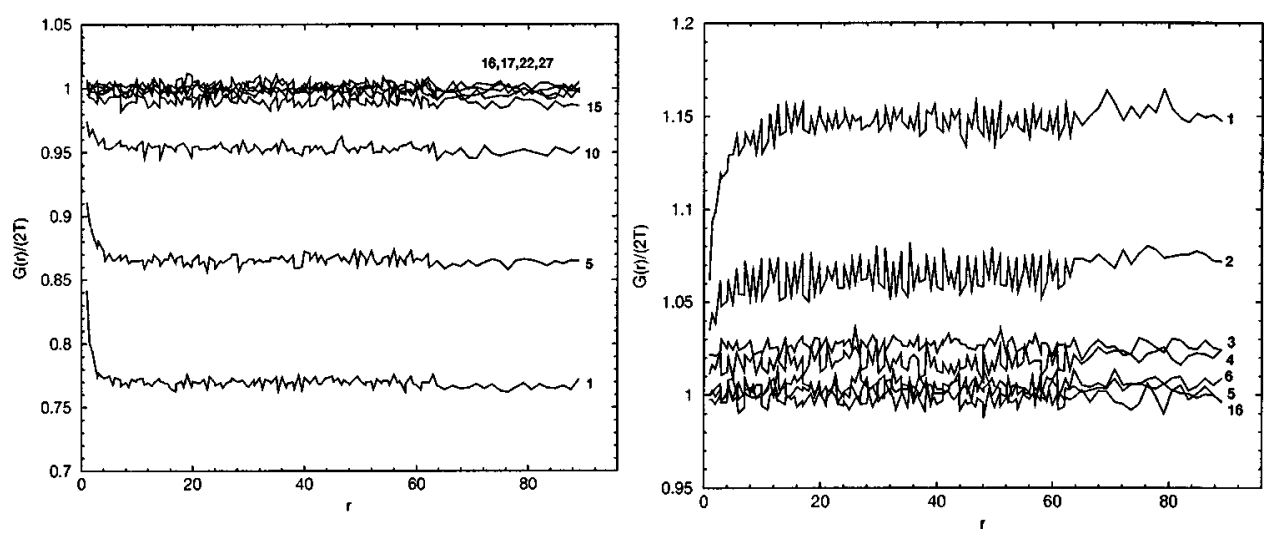

FIG. 4. Slope correlation functions (scaled by $T$ ) vs $\ln r, r$ being the distance, for the OSGM (left panel) and disordered (right panel) sine-Gordon model. Temperatures are indicated at the right side of the plots. The curve marked with temperature 16 in the plot for the RSGM is the correlation function for the OSGM at that temperature, showing clearly that both overlap.

tion for the RSGM is approximately a squared logarithm, as predicted by RG calculations. We postpone discussion of this point to the next section.

Finally we studied another magnitude, namely,

$$
m_{1}=\left\langle\cos \left[h(\mathbf{r})-h^{(0)}(\mathbf{r})\right]\right\rangle \text {. }
$$

For the OSGM this is the average computed in the preceding section, whereas for the RSGM it is the average of the cosine of the height referred to the substrate. Figure 5 shows our results: The high-temperature approximation, Eq. (24), agrees very accurately with the simulations for temperatures above $T_{R}$ (OSGM) and $T_{\mathrm{SR}}$ (RSGM). The results for both models are again indistinguishable for temperatures above $T_{R}$. Interestingly, $m_{1}$ is largely independent not only of the system size, but also on the realization of the quenched disorder for the RSGM.

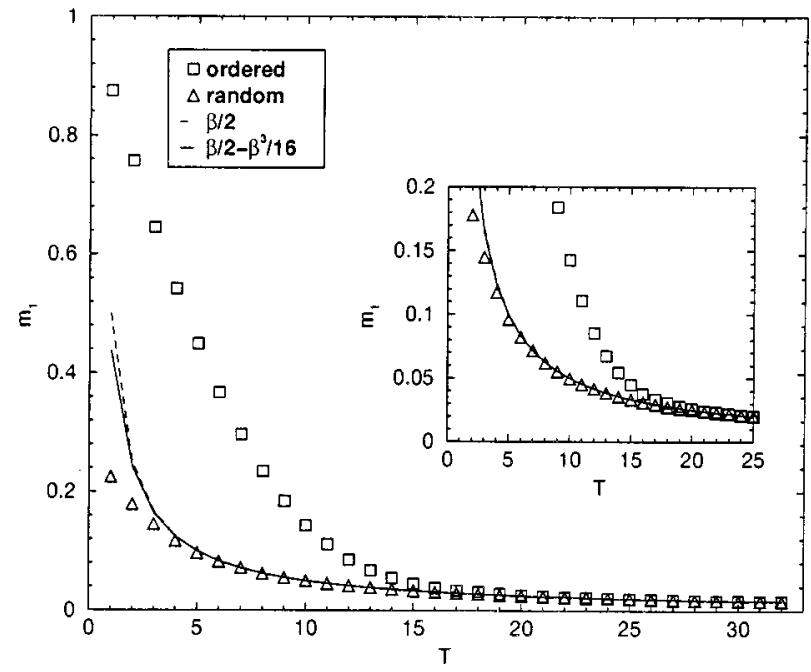

FIG. 5. Comparison of the averages of the cosine term, $m_{1}$, of the OSGM and the RSGM at different temperatures. Symbols and lines as indicated in the plot; lines correspond to theoretical approximations up to order $\beta$ and $\beta^{3}$, whereas symbols are numerical results. Results are independent of the system size and of the realization of the disorder; error bars for thermal averages are smaller than the symbol size. The inset is an enlargement of the low $m_{1}$ region of the plot.

\section{Other potential strengths}

We now turn to the question of the influence of the potential strength on the RSGM behavior. We have considered two representative values, $V_{0}=0.2$ and 5 , i.e., five times smaller and larger, respectively, than the "canonical" value $V_{0}=1$. The smallest value is close to that considered in [5], $V_{0}=0.15$, and we expect that our results will be comparable to theirs. As before, we begin by discussing the total roughness and the specific heat (see Fig. 6). First of all, for all values of $V_{0}$ there is a temperature above which the roughness value is independent of $V_{0}$ and of the presence or absence of disorder. This means that our identification of this regime with the effective suppression of any potential effect is indeed correct: Different $V_{0}$ leads only to different transition temperatures. Thus, for the OSGM we find $T_{R}^{V_{0}=02}$ $=13 \pm 1$ and $T_{R}^{V_{0}=5}=19 \pm 1$, in agreement with the intuitive expectation that larger potentials need higher temperatures to be suppressed. Aside from this, the general shape of the roughness curve is basically the same for the three values of $V_{0}$. The situation (for the OSGM) is the same as far as the specific heat is concerned: Larger (smaller) $V_{0}$ leads to larger (smaller) anomalies, which are displaced to higher (lower) $T$ following the corresponding $T_{R}$. Therefore, we conclude that changing $V_{0}$ does not introduce anything qualitatively new in the OSGM.

The picture for the RSGM is substantially different: Modifying $V_{0}$ does give rise to qualitatively new phenomena. Let us first look at the small $V_{0}$ case. Figure 6 shows that the roughness follows a straight line all the way down to $T=0$ (although we cannot exclude that there are nonlinear effects for $T \lesssim 1$ with our present resolution). This would suggest that there is no transition in this case, very much like the results of Batrouni and Hwa [5]. The upper panel of Fig. 7 , where the height-difference correlation function is depicted, confirms this interpretation, showing no dependence on temperature in the analyzed range; Fig. 8 , for $m_{1}$, agrees with this as well, insofar as the dependence of $m_{1}$ on temperature is well described by the high-temperature expansion. In view of this, we can conclude that if there is a transition, it occurs at a temperature smaller than $T \simeq 1$.

Finally, let us consider the large $V_{0}$ case, $V_{0}=5$. The plot of the roughness in Fig. 6 exhibits a striking peak for $T$ 

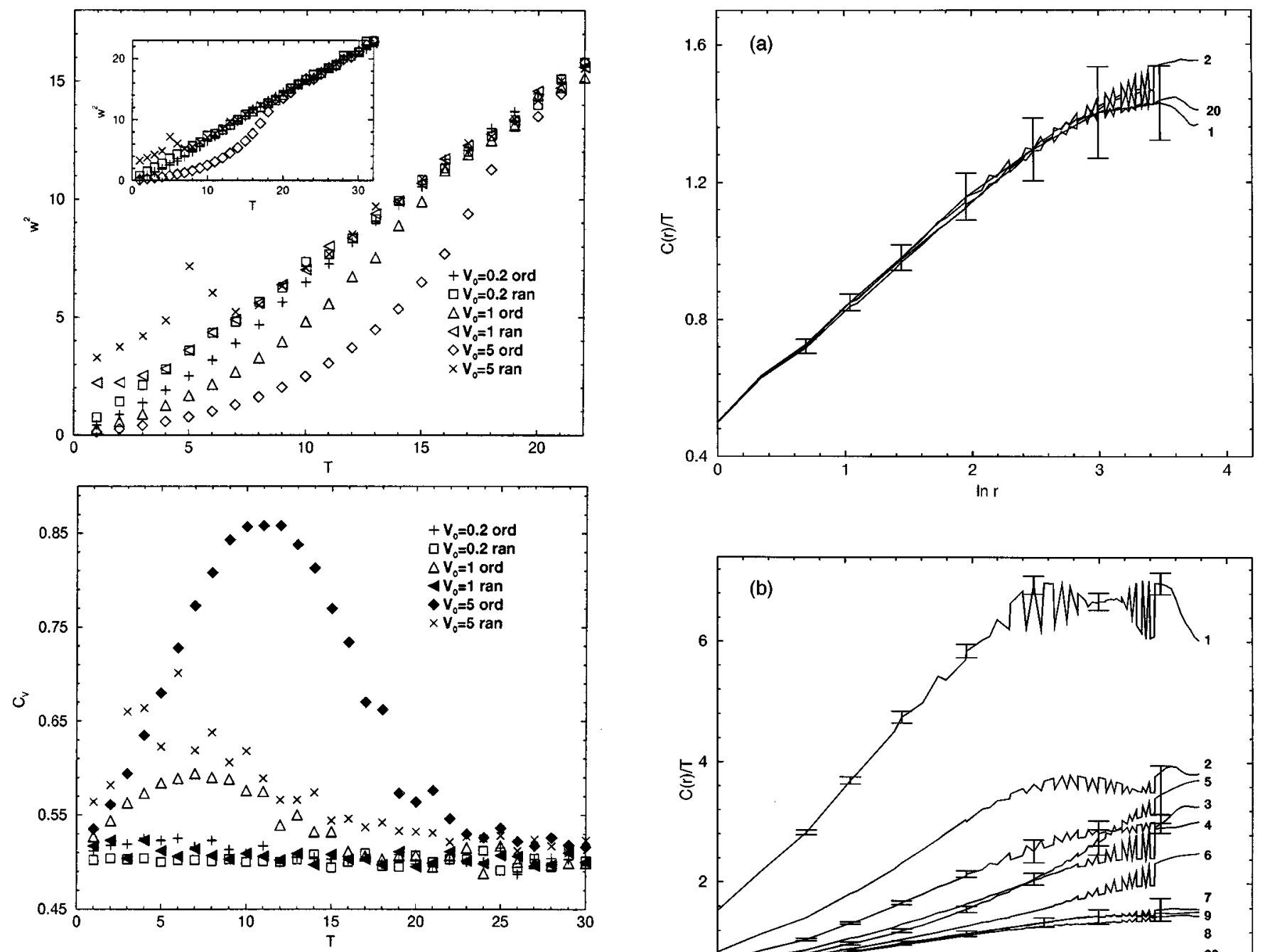

FIG. 6. Comparison of the roughness (upper panel) and specific heat (lower panel) for the OSGM and the RSGM for different values of $V_{0}$. The inset in the upper panel shows a larger range of temperatures for the roughness dependence. Symbols as indicated in the plot. All the results have been obtained for $L=64$. Note especially the peak in the specific heat for the RSGM with $V_{0}=5$.

$=5$, after which the roughness decreases until reaching the high-temperature regime (marked by the corresponding OSGM with the same value for $V_{0}$ ) at $T=8 \pm 1$. To assess the relevance of this peak in the roughness, we repeated our simulations for $L=64$ and performed additional ones for $L$ $=128$. The results, collected in Fig. 9, show that the peak is a realization-dependent feature. However, in this plot we also see that for temperatures below $T=5$ the roughness is roughly independent of the system size, something which we did not observe when $V_{0}=1$ (the lines for $V_{0}=1$ are included in Fig. 9 again for comparison). Hence, even if the peak at $T=5$ does not actually exist, that temperature does seem to separate two different regions. In addition, for $V_{0}$ $=5$ the specific heat has a (more smeared) maximum at about the same temperature as that of the roughness, although our data are much noisier and we cannot establish clearly the maximum temperature; the dependence, however, is manifestly nonmonotonic. Figure 8 supports our conclusion that $T_{\mathrm{SR}}^{V_{0}=5}=8 \pm 1$, whereas nothing special is seen as $m_{1}$ goes through $T=5$, the roughness maximum. The most

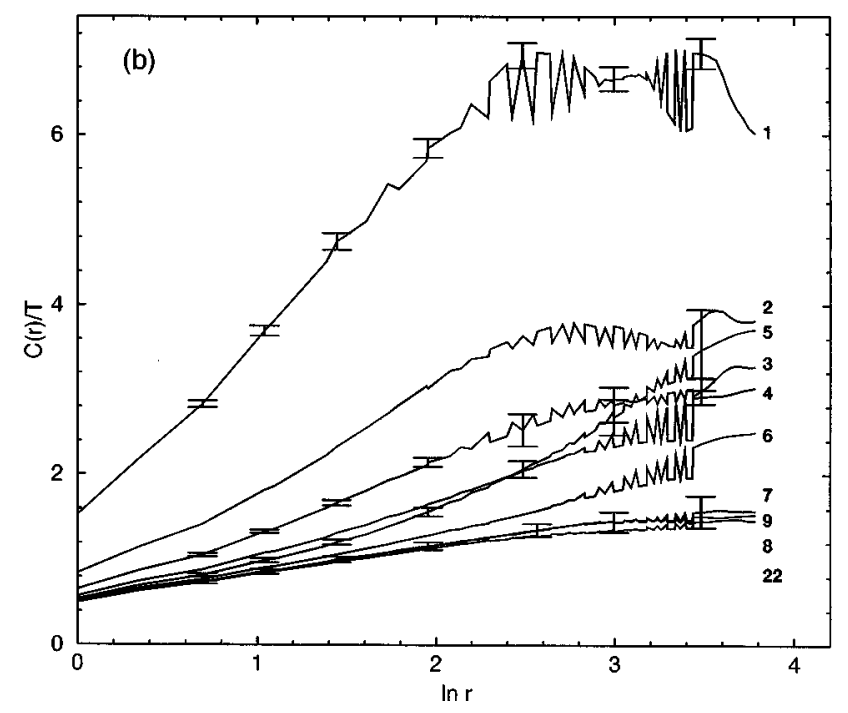

FIG. 7. Comparison of the height difference correlation functions for the RSGM with $V_{0}=0.2$ (upper panel), and $V_{0}=5$ (lower panel). Temperatures as indicated in the plots.

intriguing result is the one in Fig. 7 for the height-difference correlation function: For $T \leqslant 5$, the scaled correlations decrease with temperature but, simultaneously, the correlation length increases, up to $T=5$, when it increases beyond the system size. Above that temperature, it follows the same evolution as the $V_{0}=1$ case, finally reaching $T_{\mathrm{SR}}^{5}=8 \pm 1$. Whereas in this intermediate temperature regime the heightdifference correlation functions are well described by squared logarithms, Fig. 7 immediately shows that the lowest-temperature correlations can by no means be considered squared logarithms. This suggest the presence of a new phase transition at $T^{*}=5 \pm 1$. We will discuss the possible nature of the low-temperature phase and the existence of this transition in the next section.

\section{Other disorder strengths}

We now generalize the RSGM and let $h^{(0)}(\mathbf{r})$ be randomly chosen from a uniform distribution in $[0, \epsilon]$, with 0 $<\epsilon<2 \pi ; \epsilon=2 \pi$ is the case studied in the preceding section. 


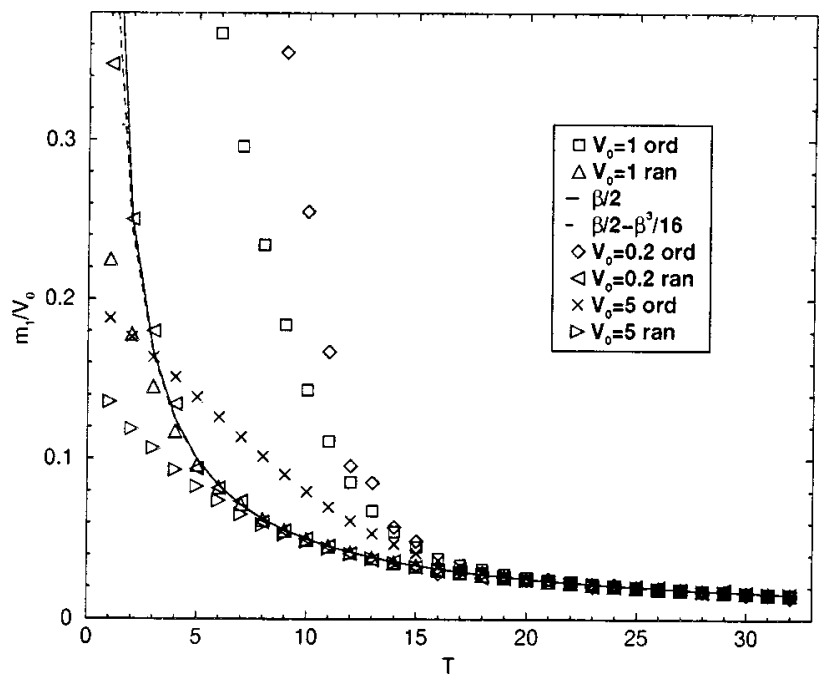

FIG. 8. Comparison of the averages of the cosine term, $m_{1}$, of the OSGM and the RSGM for different values of $V_{0}$. Symbols as indicated in the plot, and lines correspond to the theoretical approximations for high temperature.

As representative examples we have considered $\epsilon=0.8$ and 0.2 , closer to the RSGM and the OSGM, respectively. From the roughness dependence on temperature, shown in Fig. 10, we see that for both values of $\epsilon$ the dependence of the roughness on temperature is qualitatively similar to the OSGM, the results for the lower $\epsilon$ value being practically the same as for the $\epsilon=0$ case. However, the case $\epsilon=0.8$ is somewhat different: The low-temperature region appears to consist of two straight lines, changing slope at a temperature around $T^{\prime}$ $=5$, rather than a nonlinear dependence. By reasoning as above, we identify $T_{\mathrm{SR}}^{\epsilon=02} \simeq T_{R}=16 \pm 1$ and $T_{\mathrm{SR}}^{\epsilon=8}=12 \pm 1$, values which are confirmed by the energy behavior (not shown), the height difference (Fig. 11), and slope (not shown) correlation functions, and by the dependence of $m_{1}$ on temperature (not shown).

An interesting question arises from Fig. 11: There is no evidence about the squared logarithmic behavior found for

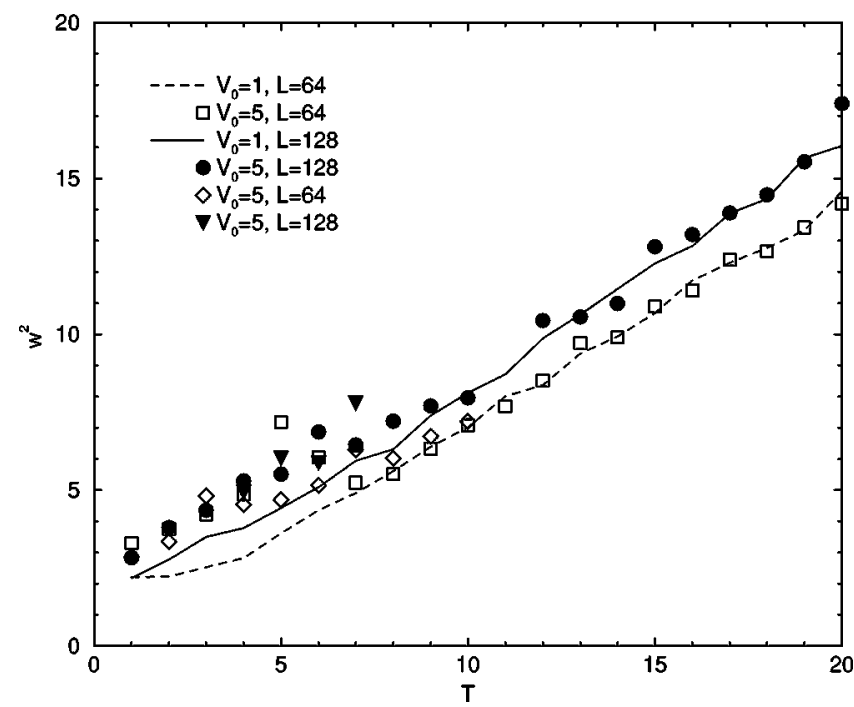

FIG. 9. Comparison of the roughness OSGM and the RSGM for $V_{0}=1,5$ and sizes $L=64,128$. Symbols as indicated in the plot. the RSGM and, furthermore, the plots exhibit a finite correlation length below $T_{\mathrm{SR}}$ for both values of $\epsilon$. Another intriguing fact is the nonmonotonic dependence of the correlation function on temperature for $\epsilon=0.8$ : From the curve for $T$ $=1$, the scaled correlation function decreases up to $T=5$; upon further increasing the temperature, the evolution of the curves is very similar to that of the OSGM. This might be connected with the change in slope in the roughness curve mentioned in the preceding paragraph (see Fig. 10), but we have not been able to draw a clearer connection. All this is clear evidence that the behavior of the RSGM is significantly dependent on the disorder strength.

\section{DISCUSSION AND CONCLUSIONS}

Let us begin the discussion of the above results by analyzing our findings about the OSGM. Our simulations strongly support that $T_{R}=16 \pm 1$ for the OSGM on a lattice with $V_{0}=1$. This is in contrast to the claims in $[39,40]$ that $T_{R}=25$ where a different way of definining the transition, which assumes the validity of the RG approach, was used (see Sec. III B and $[39,40,45]$ ). Further, the result is also in contrast with the RG prediction itself [29-31,33,34], which in our units is $T_{R}=8 \pi$. However, we believe that the comparison with the linear theory for the EW high-temperature phase has a much more physical character while keeping the basic RG ideas, and establishes beyond a doubt that for the studied lattices the roughening temperature is $T_{R}=16 \pm 1$ for $V_{0}=1$. Another hint in favor of our claim is the finding of the (Schottky) anomaly in the specific heat, which should appear below the transition temperature in view of what occurs for the $X Y$ and related models [46]. Finally, the fact that we obtain the same results for both the OSGM and the RSGM above $T_{R}$ is clear evidence that the potential is irrelevant (in the RG sense) in that regime and that we have indeed located the transition. Clearly, we cannot exclude the possibility that working on even larger lattices we would find the transition where the RG predicts it, but the absence of any finite-size effects even for $L=256$ makes this possibility quite unlikely. Another possible reason for the discrepancy is the fact that our simulations are intrinsically discrete in space while RG theories for the OSGM are always applied to the continuum equation; again, very much larger lattices would remove this objection and clarify the effects of discreteness. Aside from that, we have also found that increasing (decreasing) $V_{0}$ increases (decreases) the roughening temperature: In the cases we studied, we found $T_{R}^{V_{0}=02}=13 \pm 1$ and $T_{R}^{V_{0}=5}$ $=19 \pm 1$, which is intuitively reasonable as larger potential barriers require larger temperatures for the surface to overcome them. On the other hand, RG calculations are perturbative in $V_{0}$, so one would expect better agreement with the RG prediction for $V_{0}=0.2$, but in fact the agreement is worse in that case.

Let us now turn to the RSGM. In the "canonical" case, $V_{0}=1$, we found a super-roughening transition at $T_{\mathrm{SR}}=4$ $\pm 1=T_{R} / 4$, to be compared to RG predictions that it should occur at $T_{R} / 2$. Below $T_{\mathrm{SR}}$, we have obtained a $\ln ^{2}$ dependence of the height-difference correlation function, in agreement with RG results. However, we have clearly shown that the super-roughening transition temperature depends on $V_{0}$, confirming the earlier report by Batrouni and Hwa [5] on the 


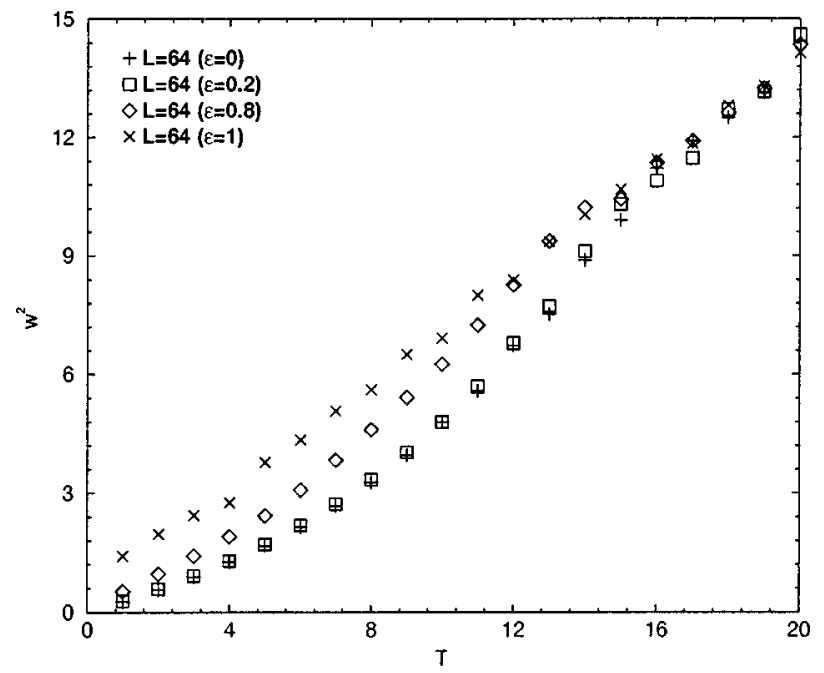

FIG. 10. Comparison of the roughness for the OSGM, the RSGM, and the two intermediate versions of the RSGM with disorder strength $\epsilon=0.2$ and 0.8 (see text for the corresponding definition). The size is $L=64$ in all four cases, and symbols are as indicated in the plot.

absence of the transition in Langevin dynamics simulations for $V_{0}=0.15$, and later reports by Rieger [6] and RuizLorenzo [18], who also observed this dependence in Monte Carlo simulations. In the opposite case, $V_{0}=5$, we find that $T_{\mathrm{SR}}^{V_{0}=5}=8 \pm 1$, considerably higher than the $V_{0}=1$ temperature. This disagrees with the RG predictions of a universal $T_{\mathrm{SR}}$ independent of $V_{0}$. We believe that the agreement between our results and the previous ones $[5,6,18]$ indeed supports a dependence of $T_{\mathrm{SR}}$ on $V_{0}$, whose explanation remains an open question as far as RG is concerned.

A second, novel finding arises when considering our numerical results for $V_{0}=5$, which strongly suggest the possibility of two different low-temperature phases. In our comments in the preceding paragraph, we took $T_{\mathrm{SR}}^{V_{0}=5}=8 \pm 1$ interpreting that the super-roughening transition implies a change from a $\ln ^{2}$ behavior of the height-difference correlation function to a ln form (and the rest of the EW features). However, the lack of size dependence of the roughness and the specific heat on temperature below $T^{*}=5 \pm 1$, with peaks absent for smaller values of $V_{0}$, raise the possible existence of another phase transition. If one looks at the correlation functions in Fig. 7, it turns out that for temperatures below $T^{*}=4$ the correlation length is finite, in agreement with the roughness independence on the system size. Whereas the range of correlations above $T^{*}$, which we believe is infinite, could be a subject of debate as we only have studied sizes up to $L=256$, our claim of finite correlation lengths below $T^{*}$ is difficult to dispute. Further evidence in this regard is shown in Fig. 12, where curves for $V_{0}=5$ at $T=1$ are compared for two different system sizes. At this point, it is interesting to recall that in a previous paper [17] it was found that at $T$ $=0$, the RSGM with $V_{0}=1$ exhibits a finite correlation length of about 20 lattice units (the reader may find Figs. 2 and 3 of [17] illustrative). Having this in mind, it is not unreasonable to conjecture that there is a $T^{*}$ for the $V_{0}=1$ case, which could be below $T=1$ or close to 1 (the upper curve in Fig. 3 might already show a finite correlation length). We stress that this phase has not been previously
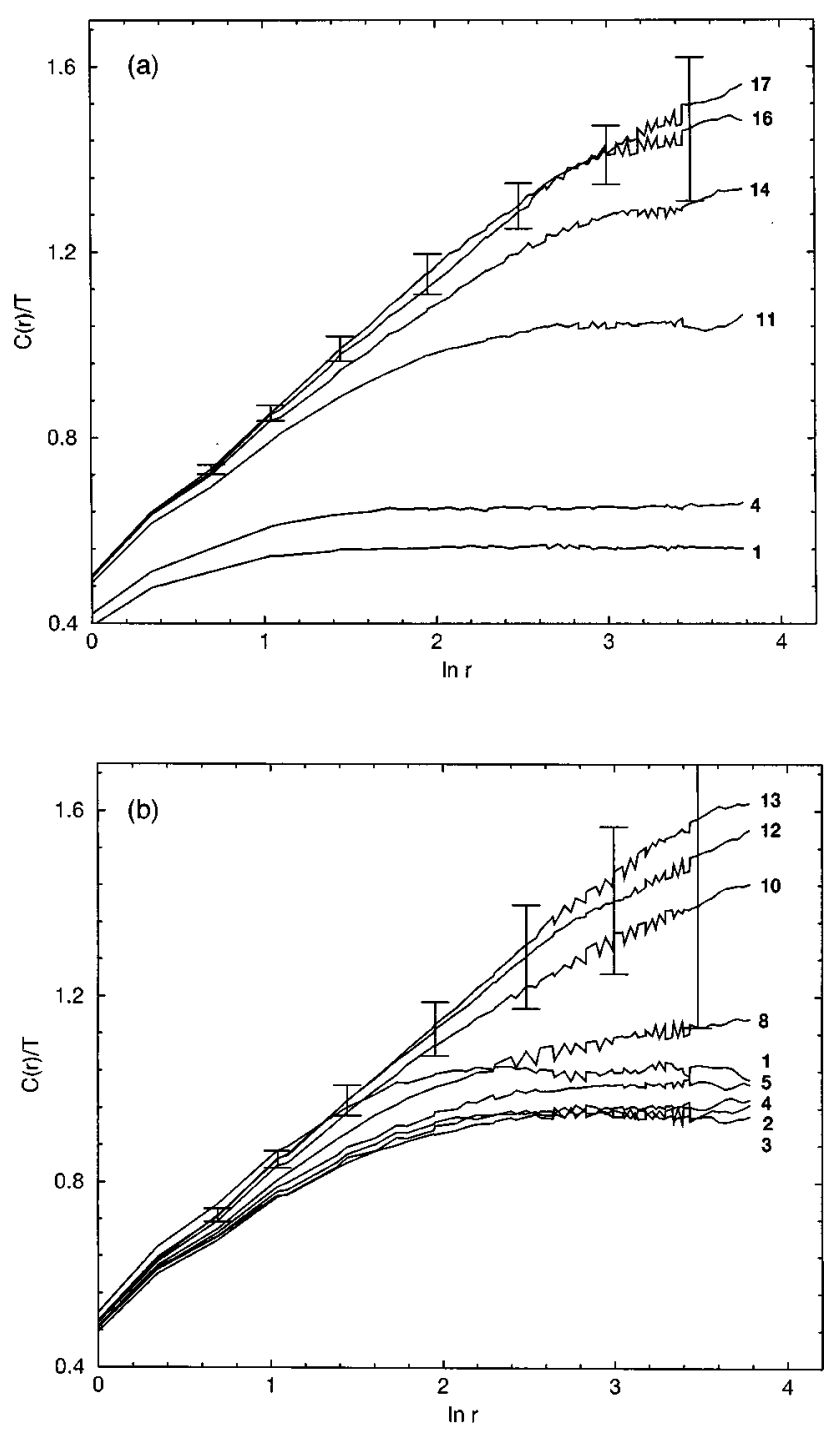

FIG. 11. Height difference correlation functions (scaled by $T$ ) vs $\ln r, r$ being distance, for the $\epsilon=0.2$ (upper panel) and $\epsilon=0.8$ (lower panel) RSGM. Temperatures are indicated at the right side of the plots.

reported in works at $T=0$ : Thus, Zeng et al. [13] studied a discrete model but, being different from the Gaussian, their low-temperature results cannot be compared to ours, and the results of Rieger et al. $[15,19]$ do not allow us to conclude anything in this respect. Intuitively, one can expect a finite correlation length phase at low temperatures and large $V_{0}$; in the limit $V_{0} \rightarrow \infty$, the surface follows the disorder [i.e., $h(\mathbf{r})=h^{(0)}(\mathbf{r})+2 n(\mathbf{r}) \pi$ everywhere], but the gradient term smoothes out the lack of correlations of $h^{(0)}(\mathbf{r})$, the competition of these two effects yielding a finite correlation length. In a loose sense, this could be interpreted as Anderson localization taking over the coupling between neighboring sites with increasing $V_{0}$. This picture is confirmed by simulations for $V_{0}=25$ (Fig. 12): For such a large value of $V_{0}$ the correlation length is only one lattice unit, i.e., correlations reach only nearest neighbors. Clearly, the data presented here are not conclusive, but the conjecture that there are two transitions whose critical temperatures depend on $V_{0}$ is not unreasonable and deserves further consideration. 


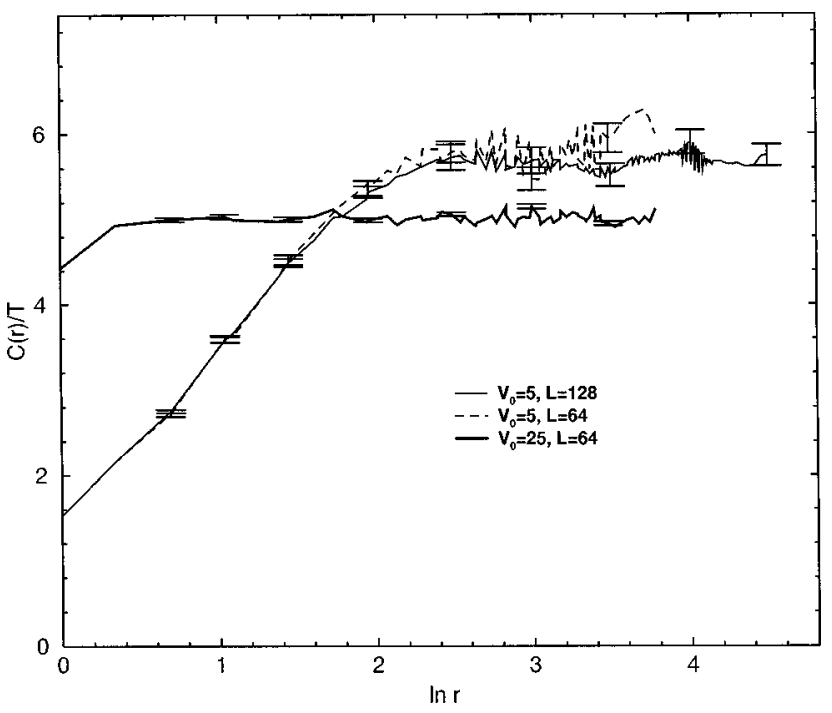

FIG. 12. Height difference correlation functions (scaled by $T$ ) vs $\ln r, r$ being distance, for different values of $V_{0}$ and $L$, as indicated in the plot. In all cases, $T=1$.

We have also found that the transition temperature and the correlation functions depend on the disorder strength. This is not unexpected, insofar as the change in the disorder distribution interacts with the periodicity of the sine potential, and therefore it is clear that when $\epsilon=2 \pi$, i.e., in the standard RSGM, it is a special case. In this respect, our results appear to indicate that the RSGM (with $\epsilon=2 \pi$ ) is a very specific model, and that its behavior at low temperatures might not be representative of what one would find in an actual experiment, where the disorder cannot be so precisely controlled. Another conclusion we may draw from our work is that there might be two classes of behavior at low temperatures for small and large $\epsilon$ : Small $\epsilon$ models would behave very similarly to the OSGM, whereas large values of $\epsilon$ would give rise to a more complex phenomenology with, e.g., nonmonotonic behavior of the correlation functions.

As a final conclusion, we remark that the most relevant result of the present work is the determination of the transition temperature from the high-temperature phase to the lowtemperature phase (or phases) of both the OSGM and the
RSGM. This poses a number of questions to be addressed either with greater numerical capabilities or with new analytical tools. We believe that the complex phase diagram of the RSGM is being partially unveiled, as our research supports previous findings such as the nonuniversality of the transition temperature. Significantly, once we know where to look for the low-temperature phase of the RSGM, we can investigate the nature of that phase (or phases). It is often stated that the RSGM is "glassy" in this regime; however, this assertion has never been really proven nor fully detailed and, furthermore, if the RG picture is qualitatively correct, it has to be recalled that it is a replica-symmetric theory, which implies that the super-rough phase would not be glassy in the replica sense [18]. We have obtained preliminary evidence that there are long-lived metastable states in the lowtemperature phase of the RSGM [47], but in view of our present results and their nonuniversality we will examine this question more carefully in future work. Investigation of the dynamics of the RSGM will also be important; we recall that Batrouni and Hwa [5] did find evidence for a superroughening transition in the dynamics of the model, and hence it would be worth checking their results for larger values of $V_{0}$. We hope that those analyses, along with measurements of nonlinear susceptibilities and of relaxation dynamics, will shed light on this difficult problem. Work along these lines is in progress.

Note added in proof. Recently, it has been drawn to our attention that, contrary to what we stated in the conclusions, there is at least one RG calculation [10] that predicts that $T_{\mathrm{SR}}$ increases if the strength of the potential increases in agreement with the numerical results reported here. We thank S. Scheidl for pointing this out to us.

\section{ACKNOWLEDGMENTS}

We thank Rodolfo Cuerno, Juan Jesús Ruiz Lorenzo, and Raúl Toral for discussions. Work at Leganés was supported by Project No. PB96-0119. Work at Los Alamos was done under the auspices of the U.S. Department of Energy. A.S. and E.M. acknowledge the support by NATO CRG 971090, by DGESIC (Spain) through an "Acción Integrada HispanoBritánica," and by Los Alamos National Laboratory for a stay at Los Alamos, where part of this work was carried out.
[1] M. Plischke and B. Bergersen, Equilibrium Statistical Physics (World Scientifc, Singapore, 1994), Chap. 11 and references therein.

[2] Spin Glasses and Random Fields, edited by A. P. Young (World Scientific, Singapore, 1997).

[3] J. Toner and D. P. DiVincenzo, Phys. Rev. B 41, 632 (1990).

[4] Y.-C. Tsai and Y. Shapir, Phys. Rev. Lett. 69, 1773 (1992); Phys. Rev. E 50, 3546 (1994); 50, 4445 (1994).

[5] G. G. Batrouni and T. Hwa, Phys. Rev. Lett. 72, 4133 (1994).

[6] H. Rieger, Phys. Rev. Lett. 74, 4964 (1995).

[7] D. Cule and Y. Shapir, Phys. Rev. Lett. 74, 114 (1995); Phys. Rev. B 51, R3305 (1995); Phys. Rev. E 53, 1553 (1996); J. Phys. A 29, 21 (1996).

[8] D. Cule, Phys. Rev. E 52, R1 (1995).

[9] J. Krug, Phys. Rev. Lett. 75, 1795 (1995).
[10] S. Scheidl, Phys. Rev. Lett. 75, 4760 (1995).

[11] E. Marinari, R. Monasson, and J. J. Ruiz-Lorenzo, J. Phys. A 28, 3975 (1995).

[12] D. J. Lancaster and J. J. Ruiz-Lorenzo, J. Phys. A 28, L577 (1995).

[13] C. Zeng, A. A. Middleton, and Y. Shapir, Phys. Rev. Lett. 77, 3204 (1996).

[14] Y.-C. Tsai, Physica A 241, 549 (1997).

[15] U. Blasum, W. Hochstättler, U. Moll, and H. Rieger, J. Phys. A 29, L459 (1996); H. Rieger and U. Blasum, Phys. Rev. B 55, R7394 (1997).

[16] B. Coluzzi, E. Marinari, and J. J. Ruiz-Lorenzo, J. Phys. A 30, 3771 (1997).

[17] A. Sánchez, A. R. Bishop, D. Cai, N. Grønbech-Jensen, and F. Domínguez-Adame, Physica D 107, 325 (1997). 
[18] J. J. Ruiz-Lorenzo, in Proceedings of the VIII Spanish Meeting on Statistical Physics FISES '97, edited by J. A. Cuesta and A. Sánchez (Editorial del Ciemat, Madrid, 1998).

[19] H. Rieger, Phys. Rev. Lett. 81, 4488 (1998).

[20] R. Salazar and R. Toral, Comput. Phys. Commun. 121-122, 40 (1999).

[21] C. Zeng, P. L. Leath, and T. Hwa, Phys. Rev. Lett. 83, 4860 (1999).

[22] F. O. Pfeiffer and H. Rieger, J. Phys. A 33, 2489 (2000).

[23] M. P. A. Fisher, Phys. Rev. Lett. 62, 1415 (1989).

[24] T. Hwa, D. R. Nelson, and V. M. Vinokur, Phys. Rev. B 48, 1167 (1993).

[25] G. Blatter, M. V. Feigel'man, V. B. Geshkenbein, A. I. Larkin, and V. M. Vinokur, Rev. Mod. Phys. 66, 1125 (1994).

[26] V. M. Vinokur and A. E. Koshelev, Zh. Eksp. Teor. Fiz. 97, 976 (1990) [Sov. Phys. JETP 70, 547 (1990)].

[27] H. Fukuyama and P. A. Lee, Phys. Rev. B 17, 535 (1978).

[28] J. Villain and J. F. Fernández, Z. Phys. B 54, 139 (1984).

[29] J. D. Weeks and G. H. Gilmer, Adv. Chem. Phys. 40, 157 (1979); J. D. Weeks, in Ordering in Strongly Fluctuating Condensed Matter Systems, edited by T. Riste (Plenum, New York, 1980).

[30] H. van Beijeren and I. Nolden, in Structure and Dynamics of Surfaces, edited by W. Schommers and P. von Blackenhagen, Topics in Current Physics Vol. 43 (Springer, Berlin, 1987).

[31] A. L. Barabási and H. E. Stanley, Fractal Concepts in Surface Growth (Cambridge University Press, Cambridge, 1995).

[32] J. Krug, Adv. Phys. 46, 139 (1997).

[33] A. Pimpinelli and J. Villain, Physics of Crystal Growth (Cam- bridge University Press, Cambridge, 1998).

[34] S. T. Chui and J. D. Weeks, Phys. Rev. B 14, 4978 (1976).

[35] H. J. F. Knops and L. W. J. den Ouden, Physica A 103, 597 (1980).

[36] N. Goldenfeld, Lectures on Phase Transitions and the Renormalization Group (Addison-Wesley, Reading, MA, 1992).

[37] J. M. Kosterlitz and D. J. Thouless, J. Phys. C 6, 1181 (1973); J. M. Kosterlitz, ibid. 7, 1046 (1974).

[38] S. F. Edwards and D. R. Wilkinson, Proc. R. Soc. London, Ser. A 381, 17 (1982).

[39] F. Falo, A. R. Bishop, P. S. Lomdahl, and B. Horovitz, Phys. Rev. B 43, 8081 (1991).

[40] A. Sánchez, D. Cai, N. Grønbech-Jensen, A. R. Bishop, and Z. J. Wang, Phys. Rev. B 51, 14664 (1995).

[41] In this context, high-temperature expansions were first used in V. Berezhinskii, Zh. Eksp. Teor. Fiz. 59, 907 (1970) [Sov. Phys. JETP 32, 493 (1971)].

[42] K. Huang, Statistical Mechanics (John Wiley and Sons, New York, 1987).

[43] H. S. Greenside and E. Helfand, Bell Syst. Tech. J. 60, 1927 (1981).

[44] M. San Miguel and R. Toral, in Instabilities and Nonequilibrium Structures $V$, edited by E. Tirapegui and W. Zeller (Kluwer, Dordrecht, 2000).

[45] W. J. Shugard, J. D. Weeks, and G. H. Gilmer, Phys. Rev. Lett. 41, 1399 (1978).

[46] C. Kawabata and A. R. Bishop, Solid State Commun. 42, 595 (1982).

[47] A. Sánchez and A. R. Bishop (unpublished). 